\title{
PERIODICITY OF FUNCTIONALS AND REPRESENTA- TIONS OF NORMED ALGEBRAS ON REFLEXIVE SPACES
}

\author{
by N. J. YOUNG \\ (Received 24th October 1974)
}

It is a well-known fact that any normed algebra can be represented isometrically as an algebra of operators with the operator norm. As might be expected from the very universality of this property, it is little used in the study of the structure of an algebra. Far more helpful are representations on Hilbert space, though these are correspondingly hard to come by: isometric representations on Hilbert space are not to be expected in general, and even continuous nontrivial representations may fail to exist. The purpose of this paper is to examine a class of representations intermediate in both availability and utility to those already mentioned-namely, representations on reflexive spaces. There certainly are normed algebras which admit isometric representations of the latter type but have not even faithful representations on Hilbert space: the most natural example is the algebra $\mathfrak{L}(E)$ of all continuous linear operators on $E$ where $E=l^{p}$ with $1<p \neq 2<\infty$, for Berkson and Porta proved in (2) that if $E, F$ are taken from the spaces $l^{p}$ with $1<p<\infty$ and $E \neq F$ then the only continuous homomorphism from $\mathfrak{L}(E)$ into $\mathfrak{L}(F)$ is the zero mapping. On the other hand there are also algebras which have no continuous nontrivial representation on any reflexive space-for example the algebra of finiterank operators on an irreflexive Banach space (see Berkson and Porta (2) or Barnes (1) or Theorem 3, Corollary 1 below).

It turns out that a normed algebra can be represented on a reflexive Banach space if and only if it admits sufficiently many weakly almost periodic functionals (Theorem 1). At this point I must record my debt and express my thanks to the referee, who pointed out to me the relevance of the technique of $\mathrm{W}$. J. Davis et al. in (14) and thereby enabled me to tidy up my results considerably by proving the more difficult implication in Theorem 1. We also obtain in the first part of the paper a number of related results on the non-existence of continuous homomorphisms between algebras through consideration of weakly almost periodic functionals. These results depend upon classifying various Banach algebras according as some, all or none of the elements of the dual are weakly almost periodic. Algebras for which the second of these three possibilities hold are said to have regular multiplication: an alternative characterisation is that the two Arens products on the bidual coincide (see (4)). This property is useful for proving results of the type described above since it enjoys 
a remarkable degree of stability: if an algebra has regular multiplication then so have all its closed subalgebras and quotient algebras. Thus, for example, knowing that every $C^{*}$-algebra has result multiplication but no infinitedimensional group algebra does (see (4) and (13) respectively) we may deduce that an infinite-dimensional group algebra cannot be isometrically isomorphic to an algebra of operators on Hilbert space with the operator norm. In order to apply this technique more generally we are led to ask which operator algebras have regular multiplication. A complete solution appears difficult, but we obtain some partial results: the algebra of finite-rank operators on a Banach space $E$ has regular multiplication if and only if $E$ is reflexive. In order that $\mathscr{I}(E)$ have regular multiplication it is thus necessary that $E$ be reflexive: we show that it is not sufficient.

In the second part we turn to the converse problem of constructing representations of algebras on reflexive spaces, and to this end introduce a stronger notion of periodicity and obtain some of its properties.

The main technical tool we use is Grothendieck's criterion of weak compactness (7, Proposition 3b). Let $E$ be a complete locally convex space with dual $E^{\prime}$ and let $\subseteq$ be a collection of $\sigma\left(E^{\prime}, E\right)$-bounded sets in $E^{\prime}$ whose $\sigma\left(E^{\prime}, E\right)$ closed absolutely convex hulls generate $E^{\prime}$ algebraically, and let the topology of $E$ agree with that of uniform convergence on the members of $\mathcal{S}$. A bounded subset $A$ of $E$ is weakly relatively compact if and only if, for each $B \in \mathcal{S}$ and each pair of sequences $\left(x_{n}\right)$ in $A$ and $\left(x_{m}^{\prime}\right)$ in $B$, the two repeated limits of the double sequence $\left(\left\langle x_{n}, x_{m}^{\prime}\right\rangle\right)$ are equal, provided they both exist. In the case of a Banach space $E$ we may take $\subseteq$ to consist of a single norm-generating subset of $E^{\prime}$.

\section{Weakly almost periodic functionals}

For this section it seems appropriate to use a more general setting than that of normed algebras. Define a locally convex semi-topological algebra to be a real or complex algebra endowed with a locally convex topology with respect to which multiplication is separately continuous, and for which $\{x y: x, y \in B\}$ is bounded for every bounded set $B$ in the algebra. A normed algebra is a real or complex algebra which is also a normed linear space and satisfies

for all elements $x, y$.

$$
\|x y\| \leqq\|x\|\|y\|
$$

Let $E$ be a locally convex space. We denote by $\mathscr{I}(E)$ the algebra of all continuous linear operators on $E$ with the topology of uniform convergence on bounded sets. A basic system of neighbourhoods of zero in $\mathfrak{L}(E)$ is given by the sets

$$
[B ; U]=\{u \in \mathfrak{L}(E): u(B) \subseteq U\},
$$

where $B$ runs through the weakly closed bounded sets of $E$ and $U$ runs through 
the closed absolutely convex neighbourhoods of zero in $E$. $\mathfrak{L}(E)$ is a locally convex semi-topological algebra. We write

$\mathfrak{R}(E)=\{u \in \mathfrak{L}(E): u(B)$ is relatively compact in $E$ for every bounded set $B$ in $E\}$ and denote by $\mathfrak{F}(E)$ the subalgebra of $\Re(E)$ comprising the operators of finite rank. Both $\mathfrak{F}(E)$ and $\mathscr{R}(E)$ will carry the topology induced by that of $\mathfrak{L}(E)$.

A representation of an algebra $\mathfrak{A}$ on a locally convex space $E$ is a homomorphism of $\mathfrak{A}$ into $\mathfrak{L}(E)$. When $\mathfrak{A}$ is locally convex we say that a representation is bounded if it maps bounded sets of $\mathfrak{A}$ onto bounded sets of $\mathfrak{L}(E)$. A representation is faithful if it is injective. If $\mathfrak{A}$ and $E$ are normed then a representation is isometric if it is an isometry with respect to the given norm of $\mathfrak{A}$ and the operator norm of $\mathfrak{Q}(E)$.

Let $\mathfrak{A}$ be a locally convex semi-topological algebra with dual $\mathfrak{U}^{\prime}$ and bidual $\mathfrak{A}^{\prime \prime}$, and let $\hat{\mathfrak{U}}^{\prime}$ be the completion of $\mathfrak{U}^{\prime}$ with respect to $\beta\left(\mathfrak{U}^{\prime}, \mathfrak{A}\right)$. Of course, in the case of normed algebras, $\hat{\mathfrak{A}}^{\prime}=\mathfrak{A}^{\prime}$. For any $h \in \mathfrak{H}^{\prime}$ and $a \in \mathfrak{A}$ we define $h a \in \mathfrak{U}^{\prime}$ by $\langle x, h a\rangle=\langle a x, h\rangle$. We shall say that a continuous linear functional $h$ on $\mathfrak{A}$ is weakly almost periodic if, for every bounded set $B$ in $\mathfrak{A}$, the set $\{h a: a \in B\}$ is $\sigma\left(\hat{\mathfrak{U}}^{\prime}, \mathfrak{A}^{\prime \prime}\right)$-relatively compact.

Lemma 1. A continuous linear functional $h$ on a locally convex semitopological algebra $\mathfrak{U}$ is weakly almost periodic if and only if, for all pairs $\left(a_{n}\right)$ and $\left(b_{m}\right)$ of bounded sequences in $\mathfrak{A}$, the two repeated limits of the double sequence $\left\langle a_{n} b_{m}, h\right\rangle$ are equal whenever they both exist.

Proof. This is a direct consequence of Grothendieck's criterion of weak compactness applied to the complete locally convex space $\hat{\mathfrak{A}}^{\prime}$.

The repeated limit criterion makes it particularly easy to see the following.

Corollary. Let $\mathfrak{A}$ and $\mathfrak{B}$ be locally convex semi-topological algebras and let $\phi: \mathfrak{A} \rightarrow \mathfrak{B}$ be a homomorphism which maps bounded sets onto bounded sets. If $h$ is a weakly almost periodic functional on $\mathfrak{B}$ then ${ }^{t} \phi h$ (where ${ }^{t} \phi$ is the adjoint of $\phi$ ) is weakly almost periodic on $\mathfrak{A}$.

Incidentally the symmetry of the condition given in Lemma 1 shows that an equivalent definition would be obtained if left-translates of $h$ were used instead of right-translates. The weakly almost periodic functionals on $\mathfrak{A}$ constitute a strongly closed subspace of $\mathfrak{A}^{\prime}$ (see (12, Theorem 2 , Corollary 1$)$ but we shall not use this fact).

Theorem 1. A normed algebra $\mathfrak{A}$ has a faithful continuous [isometric] representation on some reflexive Banach space if and only if the weakly almost periodic functionals of unit norm on $\mathfrak{A}$ separate the points of $\mathfrak{A}[$ comprise a norm-generating set]. If $\mathfrak{U}$ is a locally convex semi-topological algebra which has a faithful bounded representation on a semi-reflexive locally convex space then the weakly almost periodic functionals separate the points of $\mathfrak{A}$. 
Proof. Let $\phi: \mathfrak{A} \rightarrow \mathfrak{Q}(E)$ be a bounded monomorphism, where $E$ is a semireflexive locally convex space. For each $x \in E$ and $x^{\prime} \in E^{\prime}$ let $x \otimes x^{\prime}$ denote the continuous linear form on $\mathfrak{L}(E)$ given by

$$
\left\langle u, x \otimes x^{\prime}\right\rangle=\left\langle u x, x^{\prime}\right\rangle \text {. }
$$

We show that $x \otimes x^{\prime}$ is weakly almost periodic on $\mathfrak{L}(E)$. A simple computation shows that $\left(x \otimes x^{\prime}\right) v=x \otimes^{t} v x^{\prime}$ (where ${ }^{t} v$ is the adjoint of $v$ ). As $v$ describes a bounded set in $\mathscr{L}(E),{ }^{t} v x^{\prime}$ remains bounded in $E^{\prime}$ : we therefore need to show that $\left\{x \otimes y^{\prime}: y^{\prime} \in B^{\prime}\right\}$ is weakly relatively compact in the completion of $\mathscr{L}(E)^{\prime}$ for any bounded set $B^{\prime} \subset E^{\prime}$. It is easily seen that the mapping of $E^{\prime}$ into $\mathfrak{I}(E)^{\prime}$ given by $y^{\prime} \rightarrow x \otimes y^{\prime}$ is continuous with respect to the topologies $\beta\left(E^{\prime}, E\right)$ and $\beta\left(\mathscr{L}(E)^{\prime}, \mathfrak{L}(E)\right)$. Since any bounded set $B^{\prime}$ of $E^{\prime}$ is weakly relatively compact in $E^{\prime}$ ( $E^{\prime}$ being semi-reflexive), it follows that its image $x \otimes B^{\prime}$ in $\mathscr{L}(E)^{\prime}$ is also weakly relatively compact.

It now follows by the Corollary to Lemma 1 that ${ }^{t} \phi\left(x \otimes x^{\prime}\right)$ is a weakly almost periodic functional on $\mathfrak{U}$. Since $\phi$ is injective the functionals

$$
\left\{{ }^{t} \phi\left(x \otimes x^{\prime}\right): x \in E, x^{\prime} \in E^{\prime}\right\}
$$

separate the points of $A$; if the relevant spaces are normed and $\phi$ is isometric then $\left\{{ }^{t} \phi\left(x \otimes x^{\prime}\right):\|x\|_{E}=1=\left\|x^{\prime}\right\|_{E^{\prime}}\right\}$ is norm-generating.

The proof of the converse implication for normed algebras is deferred till the end of the paper, though it can be read straight after the definition of autocompactness at the beginning of Section 2 .

Let $\mathfrak{A}$ be a locally convex semi-topological algebra with dual $\mathfrak{U}^{\prime}$ and bidual $\mathfrak{U}^{\prime \prime}$, and regard $\mathfrak{U}$ as canonically embedded in $\mathfrak{U}^{\prime \prime}$. We shall say that $\mathfrak{A}$ has regular multiplication if the multiplication of $\mathfrak{A}$ extends to a binary operation on $\mathfrak{X}^{\prime \prime}$ which is separately continuous from $\left(\mathfrak{A}^{\prime \prime}, \sigma\left(\mathfrak{U}^{\prime \prime}, \mathfrak{A}^{\prime}\right)\right)$ to $\left(\mathfrak{U}^{\prime \prime}, \sigma\left(\mathfrak{U}^{\prime \prime}, \mathfrak{U}^{\prime}\right)\right)$, $\hat{\mathfrak{U}}^{\prime}$ denoting (as before) the completion of the strong dual of $\mathfrak{A}$. We first extend to locally convex algebras a result proved by Pym $(\mathbf{1 0}, \S 4.2)$ for Banach algebras.

Lemma 2. The following are equivalent for any locally convex semi-topological algebra $\mathfrak{A}$ :

(1) $\mathfrak{A}$ has regular multiplication;

(2) for each bounded set $B$ in $\mathfrak{A}$ and each $h \in \mathfrak{U}^{\prime}$ the function $(x, y) \rightarrow h(x y)$ on $B \times B$ has a separately $\sigma\left(\mathfrak{I}^{\prime \prime}, \mathfrak{U}^{\prime}\right)$-continuous extension to $\bar{B} \times \bar{B}$ where $\bar{B}$ is the $\sigma\left(\mathfrak{U}^{\prime \prime}, \mathfrak{A}^{\prime}\right)$-closure of $B$;

(3) every continuous linear functional on $\mathfrak{A}$ is weakly almost periodic.

Proof. Suppose the binary operation $(\xi, \eta) \rightarrow \xi \eta$ extends the multiplication of $\mathfrak{I}$ and is separately continuous from $\left(\mathfrak{I}^{\prime \prime}, \sigma\left(\mathfrak{H}^{\prime \prime}, \hat{\mathfrak{I}}^{\prime}\right)\right)$ into $\left(\mathfrak{U}^{\prime \prime}, \sigma\left(\mathfrak{A}^{\prime \prime}, \mathfrak{A}^{\prime}\right)\right)$. Then for each $h \in \mathfrak{U}^{\prime}$ the mapping $(\xi, \eta) \rightarrow\langle\xi \eta, h\rangle$ is separately $\sigma\left(\mathfrak{U}^{\prime \prime}, \mathfrak{U}^{\prime}\right)$ continuous. By Grothendieck's completeness theorem (7, page 103) a linear 
functional is $\sigma\left(\mathfrak{H}^{\prime \prime}, \hat{\mathfrak{I}}^{\prime}\right)$-continuous if and only if it is $\sigma\left(\mathfrak{H}^{\prime \prime}, \mathfrak{H}^{\prime}\right)$-continuous on $\bar{B}$ for each bounded set $B$ in $\mathfrak{A}$. Thus (1) implies (2).

Suppose, conversely, that (2) holds, and consider any pair $\xi, \eta$ in $\mathfrak{A}^{\prime \prime}$. Pick a bounded set $B$ in $\mathfrak{A}$ such that $\xi, \eta \in \bar{B}$. For each $h \in \mathfrak{H}^{\prime}$ define $\langle h, \xi \eta\rangle$ to be the value at $(\xi, \eta)$ of the separately continuous extension to $\bar{B} \times \bar{B}$ of the function $h(x y)$ on $B \times B$. This definition is independent of the particular choice of $B$, and defines $\xi \eta$ as a linear functional on $\mathfrak{A}^{\prime}$ which is clearly strongly continuous: that is, $\xi \eta \in \mathfrak{H}^{\prime \prime}$. For fixed $\xi \in \mathfrak{U}^{\prime \prime}$ the functional $\eta \rightarrow\langle\xi \eta, h\rangle$ is $\sigma\left(\mathfrak{A}^{\prime \prime}, \mathfrak{A}^{\prime}\right)$ continuous on $\bar{B}$ for every bounded set $B$ in $\mathfrak{A}$, and is therefore $\sigma\left(\mathfrak{I}^{\prime \prime}, \mathfrak{A}^{\prime}\right)$ continuous. The same applies to the mapping $\xi \rightarrow\langle\xi \eta, h\rangle$ for fixed $\eta$, and together these amount precisely to the continuity requirements of (1). Thus (2) implies (1).

It is an immediate consequence of Theorem 2 of (12) that (2) is equivalent to the following: for each pair $\left(x_{n}\right),\left(y_{m}\right)$ of bounded sequences in $\mathfrak{A}$ and each $h \in \mathfrak{A} \mathfrak{I}^{\prime}$ the two repeated limits of the double sequence $\left(h\left(x_{n} y_{m}\right)\right)$ are equal whenever they both exist. By Lemma 1 this is equivalent to (3).

When the separately continuous extension of the multiplication of $\mathfrak{A}$ to $\mathfrak{I}^{\prime \prime}$ does exist it is clearly bilinear and associative, so that $\mathfrak{U}^{\prime \prime}$ is an algebra.

Theorem 2. Let $\mathfrak{A}$ and $\mathfrak{B}$ be locally convex semi-topological algebras and let $\mathfrak{A}$ have regular multiplication. The same is true of $\mathfrak{B}$ if either $\mathfrak{B}$ is a subalgebra of $\mathfrak{A}$ (in the relative topology) or there exists a continuous algebra homomorphism $u: \mathfrak{A} \rightarrow \mathfrak{B}$ with the property that every bounded set in $\mathfrak{B}$ is contained in the closure in $\mathfrak{B}$ of the image under $u$ of a bounded subset of $\mathfrak{A}$.

Proof. When $\mathfrak{B}$ is a subalgebra of $\mathfrak{A}$ the conclusion follows from Lemma 2 and the Hahn-Banach theorem. Now suppose there exists a homomorphism $u$ with the properties described; they imply that the transpose ${ }^{t} u: \mathfrak{B}^{\prime} \rightarrow \mathfrak{U}^{\prime}$, besides being continuous, is also open with respect to the respective strong topologies, and hence the bitranspose ${ }^{t t} u$ of $u$ maps $\mathfrak{X}^{\prime \prime}$ surjectively onto $\mathfrak{B}^{\prime \prime}$. The kernel of ${ }^{t t} u$ is the annihilator in $\mathfrak{A}^{\prime \prime}$ of ${ }^{t} u\left(\mathfrak{B}^{\prime}\right)$ : we prove that it is an ideal. For each $h \in \mathfrak{U}^{\prime}$ and $y \in \mathfrak{A}$ define $y h \in \mathfrak{U}^{\prime}$ by $\langle x, y h\rangle=h(x y)$. If $h \in^{\prime} u\left(\mathfrak{B}^{\prime}\right)$ then also $y h \in{ }^{t} u\left(\mathfrak{B}^{\prime}\right)$. For any $\xi \in \mathfrak{A}^{\prime \prime}$ pick a bounded filter $\phi$ on $\mathfrak{A}$ converging to $\xi$ for $\sigma\left(\mathfrak{A}^{\prime \prime}, \mathfrak{I}^{\prime}\right)$. Since the topologies $\sigma\left(\mathfrak{H}^{\prime \prime}, \mathfrak{H}^{\prime}\right)$ and $\sigma\left(\mathfrak{H}^{\prime \prime}, \mathfrak{\mathfrak { U }}^{\prime}\right)$ agree on $\bar{B}$ for all bounded sets $B$ in $\mathscr{U}(11$, Corollary 3, p. 104), $\phi$ also tends to $\xi$ for the latter topology, and hence, using the separately continuous multiplication in $\mathfrak{A}^{\prime \prime}$, we have

$$
\langle\xi y, h\rangle=\lim _{x \rightarrow \phi}\langle x y, h\rangle=\lim _{x \rightarrow \phi}\langle x, y h\rangle=\langle\xi, y h\rangle .
$$

Thus if $\xi$ annihilates ' $u\left(\mathfrak{B}^{\prime}\right)$, so does $\xi y$ for all $y \in \mathfrak{A}$. The set

$$
\left\{\eta \in \mathfrak{A}^{\prime \prime}: \xi \eta \text { annihilates }{ }^{t} u\left(\mathfrak{B}^{\prime}\right)\right\}
$$

contains $\mathfrak{A}$ and is $\sigma\left(\mathfrak{H}^{\prime \prime}, \hat{\mathfrak{A}}^{\prime}\right)$-closed, hence contains $\bar{B}$ for every bounded set $B$ in $\mathfrak{A}$, and thus equals $\mathfrak{I}^{\prime \prime}$. $\operatorname{Ker}^{\prime \prime} u$ is therefore an ideal of $\mathfrak{I}^{\prime \prime}$, as we may obviously treat multiplication on the left similarly. 
It follows that ${ }^{~} t \boldsymbol{u}$ induces a multiplication on $\mathfrak{B}^{\prime \prime}$. To prove that this operation has the right separate continuity property write $E=\mathfrak{U}^{\prime}, F=\mathfrak{B}^{\prime}$, ${ }^{\prime} u=v$, denote the operation of left multiplication by a fixed element of $\mathfrak{A}^{\prime \prime}$ by $m$, and use the following lemma of standard type.

Lemma 3. Let $v: F \rightarrow E$ be a continuous and open linear injection of locally convex spaces and let $m:\left(E^{\prime}, \sigma\left(E^{\prime}, \hat{E}\right)\right) \rightarrow\left(E^{\prime}, \sigma\left(E^{\prime}, E\right)\right)$ be a continuous linear mapping, and suppose that the kernel of $t v$ is invariant under $m$. Then there is a unique continuous linear mapping

$$
m^{\prime}:\left(F^{\prime}, \sigma\left(F^{\prime}, \hat{F}\right)\right) \rightarrow\left(F^{\prime}, \sigma\left(F^{\prime}, F\right)\right)
$$

satisfying

$$
m^{\prime} y^{\prime}={ }^{t} v m x^{\prime} \text { for all } x^{\prime} \in E^{\prime} \text { such that }{ }^{t} v x^{\prime}=y^{\prime} .
$$

In the above $\hat{E}, \hat{F}$ are the completions of $E, F$.

In particular, if $\mathfrak{U}$ and $\mathfrak{B}$ are normed and $u: \mathfrak{U} \rightarrow \mathfrak{B}$ is continuous and relatively open and maps $\mathfrak{A}$ onto a dense subalgebra of $\mathfrak{B}$ then Theorem 2 applies. Thus a normed algebra has regular multiplication if and only if its completion does. It therefore makes no difference in the sequel if we replace the algebra $\mathfrak{F}(E)$ of finite rank operators on a Banach space $E$ by its closure in $\mathfrak{Q}(E)$.

The following lemma allows us to exploit the sequential nature of the criterion for regularity given in Lemma 2 (3).

Lemma 4. Let $K$ and $L$ be compact Hausdorff spaces and let $S(K, L)$ denote the space of separately continuous scalar functions on $K \times L$ with the topology of pointwise convergence on $K \times L$. Let $A$ be a countable set of continuous functions on $K \times L$ : if the closure $\bar{A}$ of $A$ in $S(K, L)$ is compact then every point of $\bar{A}$ is the limit of a convergent sequence of elements of $A$.

Proof. For each $f \in A$ define continuous pseudometrics $p_{f}, q_{f}$ on $K, L$ respectively by

$$
\begin{aligned}
& p_{f}\left(x, x^{\prime}\right)=\sup _{y \in L}\left|f(x, y)-f\left(x^{\prime}, y\right)\right| \\
& q_{f}\left(y, y^{\prime}\right)=\sup _{x \in K}\left|f(x, y)-f\left(x, y^{\prime}\right)\right| .
\end{aligned}
$$

Write $x \sim x^{\prime}$ if $p_{f}\left(x, x^{\prime}\right)=0$ for all $f \in A$, and let $\tilde{K}$ be the quotient space $K / \sim$ with the topology induced by the pseudometrics $p_{f}(f \in A) . \quad \tilde{K}$ is metrisable and the canonical surjection $\pi: K \rightarrow \widetilde{K}$ is continuous, so that $\widetilde{K}$ is also compact. Likewise we construct the compact metric space $L$ and quotient mapping $\rho: L \rightarrow \tilde{L}$. If $x \sim x^{\prime}$ and $y \sim y^{\prime}$ then $f(x, y)=f\left(x^{\prime}, y\right)=f\left(x^{\prime}, y^{\prime}\right)$ for all $f \in A$, and this remains true for $f \in \bar{A}$. We may therefore regard elements of $\bar{A}$ as functions on $\tilde{K} \times \tilde{L}$. Moreover, the elements of $\bar{A}$ transferred to $\widetilde{K} \times \tilde{L}$ are separately continuous on $\tilde{K} \times \tilde{L}$, and the topologies of pointwise convergence on $K \times L$ and on $\widetilde{K} \times \tilde{L}$ agree on $\bar{A}$. 
Since $\tilde{K}$ and $\tilde{L}$ are compact and metrisable they have countable dense subsets $P$ and $Q$ : consider the topology on $\bar{A}$ of pointwise convergence on $P \times Q$. This topology is Hausdorff and is coarser than the initial compact topology: it consequently agrees with the initial topology. Since $P \times Q$ is countable $\bar{A}$ is metrisable and hence has the required property.

It is worth remarking that the statement of the lemma becomes false if the hypothesis that $A$ be countable is omitted: let $K$ be the unit ball of a nonseparable Hilbert space $H$ in its weak topology, and consider the function $f(x, y)=\langle x, y\rangle$ on $K \times K$. Then $f$ is separately continuous, and is adherent to the uncountable set of continuous functions

\section{$\left\{f_{P}: P\right.$ is a projection of finite rank on $\left.H\right\}$}

where $f_{P}(x, y)=\langle P x, y\rangle$, which set is relatively compact in $S(K, K)$. However, there is no sequence in the set converging to $f$.

In order to make use of Theorems 1 and 2 we need to know about the existence or otherwise of weakly almost periodic functionals on various classes of algebra. We can give a satisfactory classification in the case of $\mathfrak{F}(E)$.

Theorem 3. Let $E$ be a locally convex space. If $E$ is reflexive then every continuous linear functional on $\mathfrak{R}(E)$ is weakly almost periodic. If $E$ is quasicomplete but not semi-reflexive then there is no non-zero weakly almost periodic functional on $\mathbb{\Im}(E)$. Thus, if $E$ is quasi-complete and barrelled, $\mathfrak{F}(E)$ has regular multiplication if and only if $E$ is reflexive.

Proof. Consider a quasi-complete space $E$ which is not semi-reflexive. $E$ contains a bounded set $B$ which is not weakly relatively compact, and hence (again by Grothendieck's criterion of weak compactness) there exists a sequence $\left(y_{m}\right)$ in $B$ and an equicontinuous sequence $\left(x_{n}^{\prime}\right)$ in $E^{\prime}$ such that the double sequence $\left(\left\langle y_{m}, x_{n}^{\prime}\right\rangle\right)$ has unequal repeated limits. Let $h$ be a non-zero continuous linear functional on $\widetilde{\mho}(E)$ : then there is a rank-one operator $y^{\prime} \otimes x \in \mathfrak{F}(E)$ such that $\left\langle y^{\prime} \otimes x, h\right\rangle \neq 0$. Introduce the rank-one operators $u_{n}=x_{n}^{\prime} \otimes x, v_{m}=y^{\prime} \otimes y_{m}$. The sequences $\left(u_{n}\right),\left(v_{m}\right)$ are bounded in $\mathscr{F}(E)$, and

$$
\left\langle u_{n} v_{m}, h\right\rangle=\left\langle y_{m}, x_{n}^{\prime}\right\rangle\left\langle y^{\prime} \otimes x, h\right\rangle,
$$

which has unequal repeated limits. By Lemma $1, h$ is not weakly almost periodic.

Suppose, conversely, that $E$ is reflexive. Recall that this implies that bounded sets of $\mathfrak{L}(E)$ are relatively compact with respect to the weak operator topology (that is, the product topology of $E_{\sigma}^{E}, E_{\sigma}$ denoting $E$ in its weak topology). Consider a continuous linear functional $h$ on $\Omega(E)$ and let

$$
A_{1}=\left(u_{n}\right), A_{2}=\left(v_{m}\right)
$$

be bounded sequences in $\Omega(E)$. Denote by $\bar{A}_{1}, \bar{A}_{2}$ the closures of $A_{1}, A_{2}$ in $\mathfrak{L}(E)$ with respect to the weak operator topology. Pick a basic neighbourhood $R(E) \cap[B ; U]$ whose polar contains $h$. 
Let $D=B \cup \bigcup_{t \in A_{2}} t(B), W=U \cap \bigcap_{t \in A_{1}} t^{-1}(U)$, and let $\bar{D}$ be the weak closure of $D$ in $E$. Since $E$ is barrelled the bounded set $\bar{A}_{1} \subset \mathfrak{L}(E)$ is equicontinuous and $W$ is therefore a neighbourhood of zero. For each $u \in \mathfrak{L}(E)$ define a function $\hat{u}$ on $\bar{D} \times W^{\circ}$ (where $W^{\circ}$ is the polar of $W$ in $E^{\prime}$ ) by

$$
\hat{u}\left(x, y^{\prime}\right)=\left\langle u x, y^{\prime}\right\rangle \quad\left(x \in \bar{D}, y^{\prime} \in W^{\circ}\right) \text {. }
$$

Give $\bar{D}$ and $W^{\circ}$ the topologies $\sigma\left(E, E^{\prime}\right)$ and $\sigma\left(E^{\prime}, E\right):$ they are then compact Hausdorff spaces, and the mapping $u \rightarrow \hat{\mathfrak{u}}$ takes $\mathscr{L}(E)$ into $S\left(\bar{D}, W^{\circ}\right)$. It is continuous with respect to the weak operator topology on $\mathscr{L}(E)$ and the pointwise topology on $S\left(\bar{D}, W^{\circ}\right)$. Furthermore, if $u \in \Omega(E)$ then the initial and weak topologies of $E$ agree on $u(\bar{D})$, so that $W^{\circ}$ is an equicontinuous set of functions on $\bar{D}$ with respect to the weak topology, and hence $\hat{a}$ is continuous on $\bar{D} \times W^{\circ}$. Let $\hat{A}_{i}=\left\{\hat{a}: u \in A_{i}\right\}$ for $i=1,2 . \hat{A}_{1}$ and $\hat{A}_{2}$ are countable sets of continuous functions on $\bar{D} \times W^{\circ}$ and their closures in $S\left(\bar{D}, W^{\circ}\right)$, being contained in the images of $\bar{A}_{1}$ and $\bar{A}_{2}$, are compact for the pointwise topology. Applying Lemma 4 we can find subsequences $\left(u_{n}\right),\left(v_{m^{\prime}}\right)$ of $A_{1}, A_{2}$ and $u, v$ in $\bar{A}_{1}, \bar{A}_{2}$ such that

$$
\lim _{n}\left\langle u_{n^{\prime}} x, y^{\prime}\right\rangle=\left\langle u x, y^{\prime}\right\rangle
$$

and

$$
\lim _{m}\left\langle v_{m^{\prime}} x, y^{\prime}\right\rangle=\left\langle v x, y^{\prime}\right\rangle
$$

for all $x \in \bar{D}, y^{\prime} \in W^{\circ}$.

We can also embed $R(E)$ in the space of all continuous functions on $B \times U^{\circ}$ in a similar manner, and (by choice of $B$ and $U$ ) $h$ is continuous with respect to the supremum norm on the image of $\Re(E)$ in $C\left(B \times U^{\circ}\right)$. By the Hahn-Banach and Riesz Representation theorems there is a regular Borel measure $\mu$ on the compact Hausdorff space $B \times U^{\circ}$ such that

for all $t \in \mathfrak{R}(E)$.

$$
\langle t, h\rangle=\int_{B \times U^{*}}\left\langle t x, y^{\prime}\right\rangle \mu\left(d x, d y^{\prime}\right)
$$

Fix any integer $m$ : for any $x \in B$ and $y^{\prime} \in U^{\circ}$ we have $v_{m} x \in D$ and $y^{\prime} \in W^{\circ}$, and hence

$$
\lim _{n}\left\langle u_{n^{\prime}} v_{m^{\prime}} x, y^{\prime}\right\rangle=\left\langle u v_{m^{\prime}} x, y^{\prime}\right\rangle
$$

By the dominated convergence theorem,

$$
\begin{aligned}
\lim _{n}\left\langle u_{n^{\prime}} v_{m^{\prime}}, h\right\rangle & =\lim _{n} \int_{B \times U^{*}}\left\langle u_{n^{\prime}} v_{m} x, y^{\prime}\right\rangle \mu\left(d x, d y^{\prime}\right) \\
& =\int_{B \times U^{*}}\left\langle u v_{m^{\prime}} x, y^{\prime}\right\rangle \mu\left(d x, d y^{\prime}\right) .
\end{aligned}
$$


Since $W^{\circ}=U^{\circ} \cup \bigcup_{t \in \lambda_{1}} t\left(U^{\circ}\right)$ (the circles denoting polars in $E^{\prime}$ ), we have also for any $x \in B$ and $y^{\prime} \in U^{\circ}$ that $x \in \bar{D}$ and ${ }^{i} u y^{\prime} \in W^{\circ}$, from which it follows that

$$
\begin{aligned}
\lim _{m}\left\langle u v_{m^{\prime}} x, y^{\prime}\right\rangle & =\lim _{m}\left\langle v_{m^{\prime}} x,{ }^{t} u y^{\prime}\right\rangle \\
& =\left\langle v x,{ }^{t} u y^{\prime}\right\rangle=\left\langle u v x, y^{\prime}\right\rangle .
\end{aligned}
$$

Applying the dominated convergence theorem again, we have

$$
\lim _{m} \lim _{n}\left\langle u_{n^{\prime}} v_{m^{\prime}}, h\right\rangle=\int_{B \times U^{\circ}}\left\langle u v x, y^{\prime}\right\rangle \mu\left(d x, d y^{\prime}\right) .
$$

Similar reasoning shows that the other repeated limit of $\left\langle u_{n^{\prime}} v_{m^{\prime}}, h\right\rangle$ has the same value. Consequently, if both repeated limits of the double sequence $\left\langle u_{n} v_{m}, h\right\rangle$ exist, they must both equal this common value. By Lemma 1 it follows that $\Re(E)$ has regular multiplication.

Corollary 1. If $E$ is a reflexive and $F$ an irreflexive Banach space there is no non-zero continuous homomorphism from $\mathfrak{F}(F)$ to $\mathfrak{L}(E)$.

Proof. If $\phi: \mathfrak{F}(F) \rightarrow \mathfrak{L}(E)$ is a continuous homomorphism then for any $x \in E$ and $x^{\prime} \in E^{\prime}$ the linear functional ${ }^{t} \phi\left(x \otimes x^{\prime}\right)$ is weakly almost periodic on $\mathfrak{F}(F)$, as shown in the proof of Theorem 1, and hence by Theorem 3

$$
{ }^{\prime} \phi\left(x \otimes x^{\prime}\right)=0:
$$

that is, $\left\langle\phi(u) x, x^{\prime}\right\rangle=0$ for all $u \in \mathfrak{F}(F), x \in E$ and $x^{\prime} \in E^{\prime}$.

Corollary 2. Let $E$ be a reflexive Banach space. If $\mathfrak{A}$ is either an infinitedimensional group algebra or the closure of $\mathfrak{F}(F)$ in $\mathfrak{L}(F)$ for some irreflexive Banach space $F$, then there is no continuous epimorphism from a closed subalgebra of $\mathfrak{R}(E)$ onto $\mathfrak{A}$.

Proof. If there were such an epimorphism then, by the open mapping theorem, it would have the boundedness property described in Theorem 2, and it would follow that $\mathfrak{A}$ had regular multiplication. However, this is not true of infinite-dimensional group algebras (13) nor of the closure of $\mathbb{F}(F)$ in $\mathscr{L}(F)$ (Theorems 2 and 3 ).

It is known that $\mathfrak{Q}(E)$ has regular multiplication if $E$ is a Hilbert space, and Theorems 2 and 3 show that if $\mathfrak{L}(E)$ has regular multiplication for some Banach space $E$ then $E$ is reflexive. It is natural to ask whether the converse holds. This is settled negatively by the following result.

Theorem 4. The group algebra $L^{1}(G)$ of any locally compact group $G$ is isometrically isomorphic to a subalgebra of $\mathfrak{L}(E)$ (with the operator norm) for some reflexive Banach space $E$. 
Proof. We consider $L^{1}(G)$ with respect to left Haar measure on $G$. Let $p_{n}=1+1 / n$ and let $E$ denote the $l^{2}$-sum of the spaces $L^{p_{n}}(G)$-that is, the space of sequences $\left(g_{n}\right)$ with $g_{n} \in L^{p_{n}}(G)$ satisfying

$$
\left\|\left(g_{n}\right)\right\|_{E}=\left\{\sum_{n=1}^{\infty}\left\|g_{n}\right\|_{p_{n}}^{2}\right\}^{+}<\infty,
$$

(where $H H_{p}$ is the norm in $L^{p}$ ). $E$ is reflexive since each $L^{p_{n}}$ is reflexive.

For $f \in L^{1}(G)$ define $T_{f} \in \mathbb{L}(E)$ by

$$
T_{f}\left(g_{n}\right)=\left(f * g_{n}\right) .
$$

Since $\|f * g\|_{p} \leqq\|f\|_{1}\|g\|_{p}$ for all $p \geqq 1$, the operator norm of $T_{f}$ is at most $\|f\|_{1}$. The mapping $f \rightarrow T_{f}$ is thus a norm-reducing homomorphism of $L^{1}(G)$ into $\mathfrak{L}(E)$. We show that it is actually an isometry.

Let $\varepsilon>0$. For each compact neighbourhood $U$ of the identity in $G$ let $e_{U}$ be a continuous non-negative function on $G$ supported by $U$ and satisfying $\left\|e_{U}\right\|_{1}=1$. Then $\left(e_{U}\right)$ is an approximate identity for $L^{1}(G)$ and hence we may find a compact neighbourhood $V$ of the identity such that

$$
\left\|f * e_{V}\right\|_{1} \geqq(1-\varepsilon)\|f\|_{1} .
$$

It suffices to prove that the representation is isometric on a dense subset of $L^{1}(G)$, so that we may assume $f$ has compact support. For any continuous function $F$ of compact support on $G,\|F\|_{p} \rightarrow\|F\|_{1}$ as $p \rightarrow 1$ by the dominated convergence theorem: we may therefore find $m \in N$ so large that

and

$$
\left\|e_{V}\right\|_{p_{m}} \leqq(1+\varepsilon)\left\|e_{V}\right\|_{1}=1+\varepsilon
$$

$$
\left\|f * e_{V}\right\|_{p_{m}} \geqq(1-\varepsilon)\left\|f * e_{V}\right\|_{1} \geqq(1-\varepsilon)^{2}\|f\|_{1} .
$$

Consider $\left(g_{n}\right) \in E$, where $g_{n}=0$ if $n \neq m$ and $g_{m}=e_{V}$ : then $\left\|\left(g_{n}\right)\right\|_{E} \leqq 1+\varepsilon$ and

$$
\left\|T_{f}\left(g_{n}\right)\right\|_{E}=\left\|f * g_{m}\right\|_{p_{m}} \geqq(1-\varepsilon)^{2}\|f\|_{1} .
$$

The operator norm of $T_{f}$ is thus at least $(1-\varepsilon)^{2}(1+\varepsilon)^{-1}\|f\|_{1}$, and therefore (since $\varepsilon$ was arbitrary) it equals $\|f\|_{1}$.

Since $L^{1}(G)$ has irregular multiplication for infinite Hausdorff $G$ (as already observed-(13)), Theorems 2 and 4 imply:

Corollary 1. Multiplication fails to be regular in $\mathfrak{Q}(E)$ for some reflexive Banach spaces $E$.

Corollary 2. There exist Banach algebras having regular multiplication whose biduals have irregular multiplication.

Proof. In the construction above take $G=Z$. Each $L^{D_{n}}(Z)$ has a basis, and consequently their $l^{2}$-sum $E$ has the approximation property. By a theorem of Grothendieck (6, Theorem 8, page 122) the bidual of $\mathbb{F}(E)$ is $\mathfrak{L}(E)$, which does not have regular multiplication; however, by Theorem $3, \mathfrak{F}(E)$ itself does have regular multiplication. 
It appears to be a difficult problem to determine for which reflexive spaces $E$ the multiplication of $\mathfrak{Q}(E)$ is regular. It is so for Montel spaces (for which $\mathfrak{L}(E)=\mathfrak{R}(E)$ ), but I do not know a wide class of reflexive Banach spaces for which it is so. The following question suggests itself: has $\mathfrak{L}(E)$ regular multiplication if $E$ is uniformly convex? Or at least if $E=l^{p}(1<p<\infty)$ ?

\section{Autoperiodic functionals}

Hitherto we have made use of weakly almost periodic functionals to prove the non-existence of continuous homomorphisms between various algebras. It would be still more useful if we could actually construct representations by means of such functionals. One might expect this to be possible, for if we are given a weakly almost periodic functional $h$ on a Banach algebra $\mathfrak{A}$, then the $w^{*}$-closure of $\{h a:\|a\| \leqq 1\}$, being absolutely convex and $w^{*}$ compact in $\mathfrak{H}^{\prime}$, miglt appear to be a potential unit ball of a reflexive space on which $\mathfrak{A}$ acts as an algebra of operators. More specifically, if we define the semi-norm $p_{h}$ on $\mathfrak{M}$ by

$$
p_{h}(x)=\sup \left\{|\langle x, h a\rangle|:\|a\|_{\mathscr{M}} \leqq 1\right\}
$$

then the completion of $\mathfrak{U} / p_{h}{ }^{-1}(0)$ is a Banach space $\mathfrak{A}_{h}$ on which the elements of $\mathfrak{A}$ act as operators in an obvious way. The trouble is that, despite the almost-periodicity of $h, \mathfrak{A}_{h}$ is not reflexive in general. For example, if $\mathfrak{A}$ is the algebra $C([0,1])$ and $h$ is the functional determined by Lebesgue measure then $h$ is weakly almost periodic (since $\mathfrak{Q}$ is a $C^{*}$-algebra) but $p_{h}$ is the $L^{1}$-norm and so $\mathfrak{X}_{h}=L^{1}[0,1]$. If we wish the above construction to work we must therefore impose a stronger condition on $h$. This leads to the following definitions.

Let $B$ be an absolutely convex subset of a real or complex vector space $E$. We denote by $E_{B}$ the quotient space $\bigcup_{\lambda>0} \lambda B / \bigcap_{\lambda>0} \lambda B$. The gauge (or Minkowski functional) of $B$, which is a seminorm on $\bigcup_{\lambda>0} \lambda B$, induces a norm \|\|$_{B}$ on $E_{B}$. We shall say that $B$ is autocompact if the unit ball of $\hat{E}_{B}$ is weakly compact, where $\hat{E}_{B}$ denotes the completion of $E_{B}$. The image of $B$ under the natural mapping of $\bigcup \lambda B$ onto $E_{B}$ is clearly dense in the unit ball of $E_{B}$.

Lemma 5. Let $\left(E, E^{\prime}\right)$ be a dual pair of vector spaces and let $B$ be a $\sigma\left(E, E^{\prime}\right)-$ closed absolutely convex subset of $E$. The following are equivalent:

(1) B is autocompact;

(2) the completion of $E_{B}$ is reflexive;

(3) for all pairs of sequences $\left(x_{n}\right)$ in $B$ and $\left(y_{m}^{\prime}\right)$ in the polar $B^{\circ}$ of $B$ in $E^{\prime}$ the two repeated limits of the double sequence $\left(\left\langle x_{n}, y_{m}^{\prime}\right\rangle\right)$ are equal whenever they both exist. 
Proof. The equivalence of (1) and (2) is just the fact that a Banach space is reflexive if and only if its unit ball is weakly compact. To prove the equivalence of (1) and (3) observe that the elements of $B^{\circ}$ induce linear functionals of norm at most one on $E_{B}$, and moreover the set of all such functionals constitutes a norm-generating subset of $E_{B}^{\prime}$, as may be shown by a straightforward Hahn-Banach argument. The equivalence thus follows from Grothendieck's criterion of weak compactness applied to $\hat{E}_{B}$.

In view of the fact that the autocompactness of $B \subseteq E$ depends on the structure of $E$ as a vector space and not on its topology it is perhaps not surprising that a closed subset of an autocompact set in a Banach space need not itself be autocompact. Nevertheless it is worth giving a cautionary example. Let $E=L^{2}[0,1]$; by Lemma 5 the unit ball of $E$ is autocompact. However, if $B$ is the closed set $\{f \in E:|f| \leqq 1$ a.e. $\}$ then $\hat{E}_{B}=L^{\infty}[0,1]$ and so $B$ is not autocompact.

We have now seen two examples of weakly compact sets in a Banach space which are not autocompact: there is, however, an implication in the reverse direction. If an absolutely convex autocompact set $B$ in a separated locally convex space $E$ is bounded and completing (this means that $E_{B}$ is complete) then $B$ is weakly relatively compact. For, since $B$ is bounded and $E$ separated, $\bigcap_{\lambda>0} \lambda B=\{0\}$, so that $E_{B}$ is a subspace of $E$. And $B$ is contained in the unit ball of $E_{B}\left(=\hat{E}_{B}\right)$, which is weakly compact for the topology of $E_{B}$, and hence also for the (coarser) initial topology of $E$.

The symmetry of condition (3) above shows that a weakly closed absolutely convex set is autocompact if and only if the same is true of its polar. This is simply a restatement of the fact that a Banach space is reflexive if and only if its dual is reflexive.

Consider now a normed algebra $\mathfrak{A}$. We shall say that $h \in \mathfrak{A}^{\prime}$ is rightautoperiodic on $\mathfrak{A}$ if the $\sigma\left(\mathfrak{U}^{\prime}, \mathfrak{2}\right)$-closure of $\left\{h a:\|a\|_{\mathfrak{U}} \leqq 1\right\}$ is autocompact in $\mathfrak{U}^{\prime}$. Likewise $h$ is left-autoperiodic if the $\sigma\left(\mathfrak{U}^{\prime}, \mathfrak{U}\right)$-closure of $\left\{\right.$ ah: $\left.\|a\|_{\mathfrak{U}} \leqq 1\right\}$ is autocompact. We call $h$ autoperiodic if it is either right- or left-autoperiodic.

Every autoperiodic functional $h$ is weakly almost periodic, for

$$
\left\{h a:\|a\|_{\mathfrak{I}} \leqq 1\right\}
$$

is strongly bounded in $\mathfrak{U}^{\prime}$ and its $\sigma\left(\mathfrak{H}^{\prime}, \mathfrak{A}\right)$-closure is $\sigma\left(\mathfrak{H}^{\prime}, \mathfrak{H}\right)$-compact and therefore completing, so that it is weakly compact by the remark above.

We now construct a representation of a normed algebra $\mathfrak{P}$ starting from an arbitrary $h \in \mathfrak{A}^{\prime}$. Let $N_{h}$ denote the subspace

$$
\{x:\langle a x, h\rangle=0 \text { for all } a \in \mathfrak{U}\}
$$

of $\mathfrak{A}$, and define a norm \|\|$_{h}$ on $\mathfrak{U} / N_{h}$ by

$$
\left\|x+N_{h}\right\|_{h}=\sup \left\{|\langle a x, h\rangle|:\|a\|_{\mathfrak{A}} \leqq 1\right\} .
$$

Let $\mathfrak{A}_{h}$ denote the completion of $\mathfrak{U} / N_{h}$ with respect to this norm. The operation of multiplication on the left by a fixed element $a \in \mathfrak{I}$ maps $N_{h}$ into itself, and 
hence induces a linear operator $a_{h}$ on $\mathfrak{A} / N_{h}$. Moreover, the operator norm of $a_{h}$ is given by

$$
\begin{aligned}
\left\|a_{h}\right\| & =\sup \left\{\left\|a x+N_{h}\right\|_{h}:\left\|x+N_{h}\right\|_{h} \leqq 1\right\} \\
& =\sup \left\{|\langle b a x, h\rangle|: x \in \mathfrak{A},\|b\|_{\mathscr{U}} \leqq 1, \sup _{\left\|b^{\prime}\right\| \mathscr{e} \leqq 1}\left|\left\langle b^{\prime} x, h\right\rangle\right| \leqq 1\right\} .
\end{aligned}
$$

If $\|a\|_{\mathfrak{M}} \leqq 1$, then for all relevant $b, b a$ is a $b^{\prime}$, and hence $\left\|a_{h}\right\| \leqq 1$. Thus $\left\|a_{h}\right\| \leqq\|a\|_{\mathfrak{A}}$ for all $a \in \mathfrak{A}$ and $h \in \mathfrak{T}^{\prime}$. Since $a_{h}$ is continuous it has a unique extension (denoted by the same symbol $a_{h}$ ) to a continuous operator on $\mathfrak{U}_{h}$. The mapping $a \rightarrow a_{h}$ is a norm-reducing representation of $\mathfrak{A}$ on the Banach space $\mathfrak{U}_{h}$.

There is also an "opposite" construction: let

$$
{ }_{h} N=\{x \in \mathfrak{A}:\langle x a, h\rangle=0 \text { for all } a \in \mathfrak{A}\}
$$

and define the norm ${ }_{h}\|\|$ on $\mathfrak{A} /{ }_{h} N$ in the obvious way. The operation of multiplication on the right by an element $a$ of $\mathfrak{A}$ induces a continuous linear operator ${ }_{h} a$ on the completion ${ }_{h} \mathfrak{A}$ of $\mathfrak{M} /{ }_{h} N$; the mapping $a \rightarrow_{h} a$ is a normreducing anti-homomorphism of $\mathfrak{U}$ into $\mathfrak{L}\left({ }_{h} \mathfrak{T}\right)$, and consequently the mapping $a \rightarrow{ }^{t}\left({ }_{h} a\right)$ is a continuous representation of $\mathfrak{A}$ on ${ }_{h} \mathfrak{H}^{\prime}$.

The idea of representing $\mathfrak{A}$ by linear operators on $\mathfrak{U} / N_{h}$ is not new: indeed when $\mathfrak{A}$ is an involutive algebra and $h$ is a positive function this is a standard piece of theory (5, IV $\S 5$ ). The difference is that $\mathfrak{U} / N_{h}$ is usually equipped with the inner product

$$
\left\langle a+N_{h}, b+N_{h}\right\rangle=\left\langle b^{*} a, h\right\rangle .
$$

The completion of $\mathfrak{X} / N_{h}$ with respect to this inner product is a Hilbert space $\mathfrak{H}_{h}$. There is a third norm we can consider on $\mathfrak{U} / N_{h}$ : the quotient norm. In general these three norms are inequivalent, as can be illustrated by the example $\mathfrak{A}=C([0,1]), h=$ Lebesgue measure. Then $N_{h}=\{0\}$ so that the quotient norm on $\mathscr{U} / N_{h}$ is the original norm. The Hilbert space norm is that of $L^{2}[0,1]$ and \|\|$_{h}$ is that of $L^{1}[0,1]$. We thus have in this case

$$
\|\|_{\mathscr{U} / N_{h}} \geqq\|\|_{\mathfrak{S}_{h}} \geqq\|\|_{h} \text {, }
$$

and this inequality remains valid (apart from positive constants) for an arbitrary $C^{*}$-algebra, and even (if the middle term is omitted) for an arbitrary Banach algebra. If $\mathfrak{A}$ is a $C^{*}$-algebra and $h$ is a positive functional the inequality

$$
\text { I }\left\|/ N_{n} \geqq\right\| h\left\|^{-1}\right\| \|_{\sigma_{h}}
$$

is well known, and, for any $x \in \mathfrak{A}$,

$$
\begin{aligned}
\left\|x+N_{h}\right\|_{h} & =\sup _{\|a\| \leqq 1}|h(a x)| \\
& \leqq \sup _{\|a\| \leqq 1} h\left(a a^{*}\right)^{\frac{1}{2}} h\left(x^{*} x\right)^{\frac{1}{t}} \\
& \leqq\|h\|^{\frac{1}{2}}\left\|x+N_{h}\right\|_{5_{h}} .
\end{aligned}
$$


And if $\mathfrak{A}$ is any Banach algebra and $h \in \mathfrak{A}^{\prime}, y \in N_{h}$ we have

$$
\begin{aligned}
\left\|x+N_{h}\right\|_{h} & =\sup _{\|a\| \leqq 1}|\langle a x, h\rangle|=\sup _{\|a\| \leqq 1}|\langle a(x-y), h\rangle| \\
& \leqq\|h\|\|x-y\|
\end{aligned}
$$

so that \|\|$_{h} \leqq\|h\|\|\|_{\mathfrak{g} / N_{h}}$.

In the case of $C^{*}$-algebras with identity, results of $\mathrm{H}$. Halpern (8) show when the three norms on $\mathfrak{U} / N_{h}$ are equivalent. Indeed, Halpern shows that the quotient and inner-product norms on $\mathfrak{A} / N_{h}$ are equivalent if and only if $h$ is a finite sum of irreducible functionals. Furthermore, for any $x \in \mathfrak{A}$,

$$
\begin{aligned}
\left\|x+N_{h}\right\|_{h} & =\sup _{\|a\| \leqq 1}|\langle a x, h\rangle| \\
& \geqq\|x\|_{\mathfrak{N}}^{-1}\left\langle x^{*} x, h\right\rangle .
\end{aligned}
$$

Since we may replace $x$ by any element of $x+N_{h}$, this yields

$$
\|\|_{h} \geqq\|\|_{\mathfrak{S}_{h}}^{2} /\|\|_{\mathscr{U} / N_{h}} \text {. }
$$

In conjunction with the inequalities already established this shows that if the quotient and inner-product norms are equivalent on $\mathfrak{U} / N_{h}$ then all three norms are equivalent. It follows from Halpern's theorem that the three norms in question are equivalent if and only if $h$ is a finite sum of irreducible functionals.

For fixed $h \in \mathfrak{U}^{\prime}$ we shall say that a set $B \subseteq \mathfrak{U}$ is left-h-bounded if

is bounded.

$$
\left\{\langle a x, h\rangle:\|a\|_{\mathscr{U}} \leqq 1, x \in B\right\}
$$

The formulation of the opposite version of the following characterisation is left to the reader.

Lemma 6. The following are equivalent for a continuous linear functional $h$ on a normed algebra $\mathfrak{U}$ :

(1) $h$ is right-autoperiodic;

(2) $\mathfrak{A}_{h}$ is reflexive;

(3) for every bounded sequence $\left(x_{n}\right)$ and left-h-bounded sequence $\left(y_{m}\right)$ in $\mathfrak{A}$ the two repeated limits of the double sequence $\left(\left\langle x_{n} y_{m}, h\right\rangle\right)$ are equal provided they both exist.

Proof. $h$ is autoperiodic if and only if the $\sigma\left(\mathfrak{A}^{\prime}, \mathfrak{A}\right)$-closure $\bar{B}$ of the set $\left\{h a:\|a\|_{\mathscr{Q}} \leqq 1\right\}$ is autocompact. By Lemma 5 this is so if and only if, for all sequences $\left(x_{n}\right)$ in the unit ball of $\mathfrak{A}$ and $\left(y_{m}\right)$ in $B^{\circ}$ (the polar of $B$ in $\mathfrak{U}$ ), the two repeated limits of $\left(\left\langle x_{n} y_{m}, h\right\rangle\right.$ are equal provided they both exist. This is clearly equivalent to condition (3).

To see that (1) and (2) are equivalent observe that $\mathfrak{A}_{h}=\hat{\mathfrak{A}}_{B^{\circ}}\left(B\right.$ and $B^{\circ}$ have their above meanings) so that $\mathfrak{A}_{h}$ is reflexive if and only if $B^{\circ}$ is autocompact, which is so if and only if its polar $\bar{B}$ in $\mathfrak{A}^{\prime}$ is autocompact-that is, $h$ is right-autoperiodic. 
Autoperiodic functionals have not nearly such good stability properties as weakly almost periodic functions. Most notably of all, the restriction of an autoperiodic functional to a closed subalgebra need not be autoperiodic. Let $E=L^{2}(0,1)$ and let $\mathfrak{U}=\mathfrak{L}(E)$ : the functional $T \rightarrow\langle T \mathbf{T}, 1\rangle$ (where 1 is the constant function equal to 1 ) is autoperiodic on $\mathfrak{A}$ (see Theorem 6 below). However, its restriction to $C([0,1])$ (which is naturally embedded in $\mathfrak{A}$, continuous functions operating on $E$ by pointwise multiplication) is not autoperiodic, as has already been observed. Nor is it true in general that the autoperiodic functionals on $\mathfrak{U}$ comprise a norm-closed subset of $\mathfrak{U}^{\prime}$, as will follow later. It is not even clear whether the sum of two right-autoperiodic functionals is right-autoperiodic. There is, however, one positive result: if $\mathfrak{A}, \mathfrak{B}$ are Banach algebras, $\phi: \mathfrak{X} \rightarrow \mathfrak{B}$ is a continuous epimorphism and $h$ is autoperiodic on $\mathfrak{B}$ then ${ }^{t} \phi h$ is autoperiodic on $\mathfrak{A}$. For suppose that $\left(a_{n}\right)$ is bounded and $\left(b_{n}\right)$ is ${ }^{t} \phi h$-bounded in $\mathfrak{A}$, so that, for some $K>0$,

$$
\left|\left\langle\phi(a) \phi\left(b_{n}\right), h\right\rangle\right| \leqq K
$$

for all $n \in N$ and $a \in \mathfrak{A}$ such that $\|a\| \leqq 1$. By the open mapping theorem $\{\phi(a):\|a\| \leqq 1\}$ contains a ball of positive radius in $\mathfrak{B}$, and hence $\left(\phi\left(b_{n}\right)\right)$ is $h$-bounded in $\mathfrak{B}$. It follows that the repeated limits of

$$
\left\langle\phi\left(a_{n}\right) \phi\left(b_{m}\right), h\right\rangle=\left\langle a_{n} b_{m},{ }^{t} \phi h\right\rangle
$$

are equal, provided they exist.

We continue our comparison of the two types of periodicity by studying the autoperiodic functionals on the operator algebras considered in Section 1. We have seen that if $E$ is reflexive then $x \otimes x^{\prime}$ is weakly almost periodic on $\mathfrak{L}(E)$ for any $x \in E, x^{\prime} \in E^{\prime}$, and the same is consequently true for the restriction of $x \otimes x^{\prime}$ to any subalgebra of $\mathscr{L}(E)$. The example given above $(1 \otimes 1$ on $\left.\mathfrak{S}\left(L^{2}(0,1)\right)\right)$ shows that some modification is needed for autoperiodic functionals.

Lemma 7. Let $E$ be a reflexive Banach space, $\mathfrak{2}$ a Banach algebra, $\pi: \mathfrak{A} \rightarrow \mathfrak{Q}(E)$ a continuous representation and $x_{1}^{\prime}, \ldots, x_{k}^{\prime} \in E^{\prime}$. If either of the equivalent conditions

(a) $\left\{\left({ }^{t}(\pi(a)) x_{1}^{\prime}, \ldots,{ }^{t}(\pi(a)) x_{k}^{\prime}\right): a \in \mathfrak{R}\right\}$ is closed in $E^{\prime} \oplus \ldots \oplus E^{\prime}$;

(b) $\left\{{ }^{t} \pi\left(x_{1} \otimes x_{1}^{\prime}\right)+\ldots+{ }^{t} \pi\left(x_{k} \otimes x_{k}^{\prime}\right): x_{1}, \ldots, x_{k} \in E\right\}$ is norm closed in $\mathfrak{U}^{\prime}$

holds, then ${ }^{t} \pi\left(x_{1} \otimes x_{1}^{\prime}\right)+\ldots+{ }^{t} \pi\left(x_{k} \otimes x_{k}^{\prime}\right)$ is right-autoperiodic on $\mathfrak{A}$ for any $x_{1}, \ldots, x_{k} \in E$.

Proof. Let $E^{k},\left(E^{\prime}\right)^{k}$ denote the direct sums of $k$ copies of $E, E^{\prime}$ respectively. To show that $(a)$ and $(b)$ are equivalent define the continuous linear mapping $L: \mathfrak{A} \rightarrow\left(E^{\prime}\right)^{k}$ by $L a=\left(^{\prime}(\pi(a)) x_{1}^{\prime}, \ldots,,^{\prime}(\pi(a)) x_{k}^{\prime}\right)$. The adjoint ${ }^{t} L: E^{k} \rightarrow \mathfrak{A}^{\prime}$ of $L$ is given by ${ }^{t} L\left(x_{1}, \ldots, x_{k}\right)={ }^{t} \pi\left(x_{1} \otimes x_{1}^{\prime}\right)+\ldots+{ }^{t} \pi\left(x_{k} \otimes x_{k}^{\prime}\right)$. The equivalence now follows from the fact that a continuous linear mapping between Banach spaces has closed image if and only if the same is true of its adjoint.

E.M.S. $-20 / 2-\mathrm{H}$ 
Suppose, then, that $F^{\circ}=\left\{\left({ }^{t}(\pi(a)) x_{1}^{\prime}, \ldots,{ }^{t}(\pi(a)) x_{k}^{\prime}\right): a \in \mathfrak{A}\right\}$ is closed in $\left(E^{\prime}\right)^{k}$. Let $F$ be the annihilator of $F^{\circ}$ in $E^{k}$ : then $F^{\circ}$ is the dual of $E^{k} / F$ (note that, on account of reflexivity, weak and norm closure are the same for subspaces of $\left.\left(E^{\prime}\right)^{k}\right)$. For each $a \in \mathfrak{A}, \pi(a) \oplus \ldots \oplus \pi(a)$ maps $F$ into itself, and therefore induces an operator $\tilde{\pi}(a)$ on $E^{k} / F$.

Now consider any $x_{1}, \ldots, x_{k} \in E$, write $h={ }^{t} \pi\left(x_{1} \otimes x_{1}^{\prime}\right)+\ldots+{ }^{t} \pi\left(x_{k} \otimes x_{k}^{\prime}\right)$, and pick an $h$-bounded sequence $\left(b_{m}\right)$ in $\mathfrak{A}$. There is some $K>0$ such that

$$
\mid\left\langle\pi\left(b_{m}\right) \oplus \ldots \oplus \pi\left(b_{m}\right)\left(x_{1}, \ldots, x_{k}\right),\left({ }^{\prime}\left(\pi(a) x_{1}^{\prime}, \ldots,{ }^{\prime}(\pi(a)) x_{k}^{\prime}\right)\right\rangle\right| \leqq K\|a\|
$$

for every $a \in \mathfrak{A}$ and $m \in N$. In other words, for every $y^{\prime} \in F^{\circ}$,

$$
\left\{\left\langle\tilde{\pi}\left(b_{m}\right) x, y^{\prime}\right\rangle: m \in N\right\}
$$

is bounded, where $x=\left(x_{1}, \ldots, x_{k}\right)+F \in E^{k} / F$. Thus the sequence $\left(\tilde{\pi}\left(b_{m}\right) x\right)$ is bounded in the reflexive space $E^{k} / F$, and so has a weak cluster point $x^{\circ}$. And if $\left(a_{n}\right)$ is any bounded sequence in $\mathfrak{U}$ then $\left\{\left({ }^{t}\left(\pi\left(a_{n}\right)\right) x_{1}^{\prime}, \ldots,{ }^{t}\left(\pi\left(a_{n}\right)\right) x_{k}^{\prime}\right): n \in N\right\}$ is bounded in $F^{\circ}$, and so has a weak cluster point $y^{\circ}$. If therefore the two repeated limits of the double sequence

$$
\left\langle a_{n} b_{m},{ }^{t} \pi h\right\rangle=\left\langle\tilde{\pi}\left(b_{m}\right),\left({ }^{t}\left(\pi\left(a_{n}\right)\right) x_{1}^{\prime}, \ldots,{ }^{t}\left(\pi\left(a_{n}\right)\right) x_{k}^{\prime}\right)\right\rangle
$$

exist, they must both equal $\left\langle x^{\circ}, y^{\circ}\right\rangle$. It follows that ${ }^{t} \pi h$ is right-autoperiodic on $\mathfrak{A}$.

In this instance it is worth stating the opposite version separately.

Lemma 7'. Let $E$ be a reflexive Banach space, $\mathfrak{A}$ a Banach algebra,

$$
\pi: \mathfrak{A} \rightarrow \mathfrak{L}(E)
$$

a continuous representation and $x_{1}, \ldots, x_{k} \in E$. If either of the equivalent conditions

(a) $\left\{\left(\pi(a) x_{1}, \ldots, \pi(a) x_{k}\right): a \in \mathfrak{A}\right\}$ is closed in $E \oplus \ldots \oplus E$;

(b) $\left\{t^{t}\left(x_{1} \otimes x_{1}^{\prime}\right)+\ldots+{ }^{t} \pi\left(x_{k} \otimes x_{k}^{\prime}\right): x_{1}^{\prime}, \ldots, x_{k}^{\prime} \in E^{\prime}\right\}$ is norm closed in $\mathfrak{I}^{\prime}$ holds, then ${ }^{t} \pi\left(x_{1} \otimes x_{1}^{\prime}\right)+\ldots+{ }^{t} \pi\left(x_{k} \otimes x_{k}^{\prime}\right)$ is left-autoperiodic on $\mathfrak{Q}$ for any

$$
x_{1}^{\prime}, \ldots, x_{k}^{\prime} \in E^{\prime} \text {. }
$$

Theorem 5. Let $\mathfrak{A}$ be a complex Banach algebra and let $\pi$ be an irreducible representation of $\mathfrak{U}$ on a reflexive Banach space $E$ (so that the only subspaces of $E$ invariant under $\pi(\mathfrak{2})$ are $\{0\}$ and $E$ ). Every element of $E \otimes E^{\prime}$ determines a left-autoperiodic functional on $\mathfrak{A}$, and if $\pi(\mathfrak{M}) \supseteq \mathfrak{F}(E)$ then this functional is also right-autoperiodic.

Proof. According to a theorem of Johnson (9) $\pi$ is necessarily continuous, so that we may apply Lemma 7. Let $h=x_{1} \otimes x_{1}^{\prime}+\ldots+x_{k} \otimes x_{k}^{\prime} \in E \otimes E^{\prime}$, where the $x$ 's and $x$ 's are linearly independent. By Lemma 7 ', ' $\pi h$ is left-autoperiodic on $\mathfrak{A}$ provided that the subspace $\left\{\left(\pi(a) x_{1}, \ldots, \pi(a) x_{k}\right): a \in \mathfrak{N}\right\}$ is closed in $E^{k}$. 
By a well-known theorem (for example $(3, \S 25)$ ) this subspace is in fact the whole of $E^{k}$. And if $\pi(\mathfrak{A}) \supseteq \mathfrak{F}(E)$ one readily checks that

$$
\left\{\left({ }^{\prime}(\pi(a)) x_{1}^{\prime}, \ldots,{ }^{t}(\pi(a)) x_{k}^{\prime}\right): a \in \mathfrak{A}\right\}=\left(E^{\prime}\right)^{k},
$$

and so by Lemma $7,{ }^{t} \pi h$ is also right-autoperiodic.

Corollary. Every irreducible representation of a Banach algebra $\mathfrak{A}$ on a reflexive Banach space arises as the natural representation of $\mathfrak{\mathfrak { l }}$ on ${ }_{h} \mathfrak{A}$ for some left autoperiodic functional $h \in \mathfrak{H}^{\prime}$.

Proof. Let $\pi: \mathfrak{A} \rightarrow \mathfrak{L}(E)$ be an irreducible representation where $E$ is reflexive. Pick any non-zero $x \in E, x^{\prime} \in E^{\prime}$ and let $h={ }^{t} \pi\left(x \otimes x^{\prime}\right)$. We show that $\pi$ is topologically equivalent (in the usual sense of representation theory) to the representation $a \rightarrow^{t}\left({ }_{h} a\right)$ of $\mathfrak{U}$ on ${ }_{h} \mathfrak{H}$. If $a \in{ }_{h} N$ then $\left\langle\pi(a b) x, x^{\prime}\right\rangle=0$ for all $b \in \mathfrak{U}$, and since $\pi(\mathfrak{Q}) x=E$ it follows that ${ }^{t}(\pi(a)) x^{\prime}=0$. We may therefore define a linear mapping $v: \mathfrak{A} /{ }_{h} N \rightarrow E^{\prime}$ by $v\left(a+{ }_{h} N\right)={ }^{\prime}(\pi(a)) x^{\prime}$. We have, moreover,

$$
{ }_{n}\left\|a+{ }_{h} N\right\|=\sup _{\|b\| \leqq 1}|\langle a b, h\rangle|=\sup _{\|b\| \leqq 1}\left|\left\langle\pi(b) x,{ }^{t}(\pi(a)) x^{\prime}\right\rangle\right|
$$

from which we may deduce (using the open mapping theorem and the fact that $\pi$ is irreducible) that $v$ is bicontinuous with respect to the norms of $\mathfrak{U} /{ }_{h} N$ and $E^{\prime}$. The adjoint mapping ${ }^{t} v: E \rightarrow_{h} \mathfrak{A}^{\prime}$ is also bicontinuous. To prove the two representations in question equivalent we show that, for every $a \in \mathfrak{U}$, the diagram

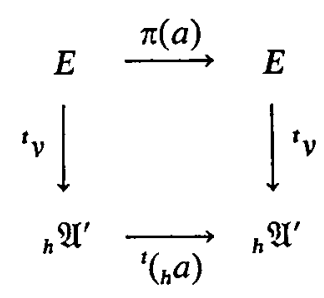

commutes: that is for all $\xi \in E$ and $b \in \mathfrak{A}$,

$$
\left\langle b+{ }_{h} N,{ }^{t} v \pi(a) \xi\right\rangle=\left\langle b+{ }_{h} N,{ }^{t}\left({ }_{h} a\right)^{t} v \xi\right\rangle .
$$

Using the fact that ${ }_{h} a\left(b+{ }_{h} N\right)=b a+{ }_{h} N$ one easily reduces both sides to $\left\langle\pi(b a) \xi, x^{\prime}\right\rangle$. By Theorem 6 (or since ${ }_{h} \mathfrak{A}=E^{\prime}$ ) $h$ is autoperiodic.

The Corollary shows that one approach to the construction of all irreducible representations of an algebra on reflexive spaces is to try to find all left-autoperiodic functionals on the algebra. Of course, this will be a difficult task in general: here is one case where it is possible.

Theorem 6. The autoperiodic functionals on $\Re(E)$, where $E$ is Hilbert space, are precisely those determined by the elements of $E \otimes E$. 
Proof. Note that, since $\mathcal{R}(E)$ is conjugate-isomorphic to its opposite algebra, we need not distinguish between right- and left-autoperiodicity. By Theorem 6 each element of $E \otimes E$ determines an autoperiodic functional on $\Re(E)$. To prove the converse we use the fact that the dual of $\Re(E)$ is the trace class of operators (see, for example, (6)). That is, if $h \in \mathcal{R}(E)^{\prime}$ there exist finite or countable orthonormal sequences $\left(e_{n}\right)$ and $\left(f_{n}\right)$ in $E$ and $\lambda_{n}>0$ such that $\Sigma \lambda_{n}<\infty$ and $h=\Sigma \lambda_{n} e_{n} \otimes f_{h}$. Suppose $h \notin E \otimes E$, so that this sum is infinite. Let $v_{m}$ be the rank-one operator $\lambda_{m}^{-1} e_{m} \otimes f_{m}$ : for any $u \in \mathfrak{R}(E)$ we have

$$
\left\langle u v_{m}, h\right\rangle=\sum_{n} \lambda_{n}\left\langle u v_{m} e_{n}, f_{n}\right\rangle=\left\langle u f_{m}, f_{m}\right\rangle \text {, }
$$

so that the sequence $\left(v_{m}\right)$ is $h$-bounded. And if $u_{n} \in \mathfrak{R}(E)$ is defined to be $\sum_{j=1}^{n} f_{j} \otimes f_{j}$ then $\left\|u_{n}\right\|=1$ and we find that

$$
\left\langle u_{n} v_{m}, h\right\rangle= \begin{cases}1 & \text { if } m \leqq n \\ 0 & \text { if } m>n .\end{cases}
$$

Lemma 6 now shows that $h$ is not autoperiodic.

This example justifies the assertion above that the set of autoperiodic functionals on $\mathfrak{U}$ need not be norm-closed in ' $\mathfrak{A}$ '.

It does not seem to be easy to describe the autoperiodic functionals on $\mathscr{L}(E)$ for $E$ a Hilbert space. The answer in this case is certainly not $E \otimes E$, for there are non-zero autoperiodic functionals on $\mathfrak{L}(E)$ which annihilate $\Re(E)$ (these may be constructed using an irreducible representation of the Calkin algebra $\mathfrak{L}(E) / \mathfrak{\Omega}(E)$ ). There is, however, one more class of $C^{*}$-algebras for which a description can be given.

Theorem 7. The autoperiodic functionals on a commutative $C^{*}$-algebra are precisely the linear combinations of characters.

Proof. Consider the commutative $C^{*}$-algebra $C(K), K$ a compact Hausdorff space. If $\mu \in C(K)^{\prime}$ is a linear combination of characters $C(K)_{\mu}$ is finitedimensional and so reflexive. Conversely, suppose $\mu$ is not a linear combination of characters: then it is easily seen that $\mu$, regarded as a regular Borel measure on $K$, has an infinite set $\left\{t_{n}\right\}$ of density points $(t \in K$ is a density point of $\mu$ if $|\mu|(U)>0$ for every neighbourhood $U$ of $t)$. Passing to a subset if necessary we may assume that $\left\{t_{n}\right\}$ is discrete-that is, pairwise disjoint neighbourhoods $\left\{U_{n}\right\}$ of the $\left\{t_{n}\right\}$ exist. By the regularity of $\mu$ there exists a compact subset $C_{n}$ of $U_{n}$ such that $|\mu|\left(C_{n}\right)>\frac{3}{4}|\mu|\left(U_{n}\right)>0$. We have $d|\mu|=g \mathrm{~d} \mu$, where $g$ is measurable and $|g|=1$ identically on $K$. By Lusin's theorem there is a continuous function $g_{n}$ on $C_{n}$ agreeing with $g$ except on a subset $D_{n}$ of $C_{n}$ of $|\mu|$-measure at most $|\mu|\left(U_{n}\right) / 8$ and satisfying $\left|g_{n}\right| \leqq 1$ on $C_{n}$. Extend $g_{n}$ to a continuous function on $K$ in such a way that $\left|g_{n}\right| \leqq 1$ on $K$ and $g_{n}=0$ on $K \backslash U_{n}$. Let $h_{n}=g_{n} /|\mu|\left(U_{n}\right)$. For any $f \in C(K)$ we have

$$
\left|\int_{K} f h_{n} d \mu\right| \leqq \int_{U_{n}}\left|f h_{n}\right| d|\mu| \leqq\|f\|\|\mu\|,
$$


so that $\left(h_{n}\right)$ is a $\mu$-bounded sequence in $C(K)$. For each $m \in N$ choose $f_{m} \in C(K)$ of unit norm so that $f_{m} \mid C_{j}=1$ for $1 \leqq j \leqq m$ and $f_{m}=0$ outside $U_{1} \cup \ldots \cup U_{m}$. If $n>m$ we have

$$
\int_{K} f_{m} h_{n} d \mu=0
$$

And if $n \leqq m$,

$$
\int_{K} f_{m} h_{n} d \mu=\frac{1}{|\mu|\left(U_{n}\right)}\left(\int_{U_{n} \backslash C_{n}}+\int_{C_{n} \backslash D_{n}}+\int_{D_{n}} f_{m} g_{n} d \mu\right)
$$

On $C_{n} \backslash D_{n}$ we have $f_{m}=1$ and $g_{n} d \mu=d|\mu|$, so that the middle integral is $|\mu|\left(C_{n}\right)-|\mu|\left(D_{n}\right)$. And estimating the outer integrals using the fact that $\left|f_{m} g_{n}\right| \leqq 1$,

$$
\begin{aligned}
\left|\int_{K} f_{m} h_{n} d \mu-\frac{|\mu|\left(C_{n}\right)}{|\mu|\left(U_{n}\right)}\right| & \leqq \frac{1}{|\mu|\left(U_{n}\right)}\left(|\mu|\left(U_{n} \backslash C_{n}\right)+2|\mu|\left(D_{n}\right)\right) \\
& <\frac{1}{2} .
\end{aligned}
$$

Since $|\mu|\left(C_{n}\right)>\frac{3}{4}|\mu|\left(U_{n}\right)$ it follows that, for $n>m$,

$$
\left|\int_{K} f_{m} h_{n} d \mu\right| \geqq \frac{1}{4}
$$

We may choose subsequences $\left(f_{m}\right)$ and $\left(h_{n}\right)$ of $\left(f_{m}\right)$ and $\left(h_{n}\right)$ so that both repeated limits of $\int_{K} f_{m^{\prime}}, h_{n^{\prime}} d \mu$ exist: one is zero and the other has absolute value at least a quarter, so that $\mu$ is not autoperiodic, by Lemma 6 .

There are several natural questions concerning autoperiodic functionals in addition to the ones already raised in the text, and some of these are related to open questions about operator algebras. We may ask whether the unpleasing asymmetry of the conclusion of Theorem 5 can be eliminated: that is, if $\pi$ is an irreducible representation of a Banach algebra $\mathfrak{A}$ on a reflexive space $E$ is it true that ' $\pi\left(x \otimes x^{\prime}\right)$ is right-autoperiodic for every $x \in E$ and $x^{\prime} \in E^{\prime}$ ? In order to construct a counter-example we must arrange that $\left\{{ }^{t}(\pi(a)) x^{\prime}: a \in \mathfrak{A}\right\}$ be not closed in $E^{\prime}$, which seems quite difficult. Another range of questions arises if we interest ourselves in continuous topologically irreducible representations of an algebra $\mathfrak{U}$ on a reflexive space $E$ (that is, representations for which the only closed subspaces invariant under $\pi(\mathfrak{2})$ are $\{0\}$ and $E)$. Is it still true that every element of $E \otimes E^{\prime}$ determines a left-autoperiodic functional on $\mathfrak{A}$ ? I do not even know the answer in the case $E$ a Hilbert space and $\mathfrak{A}$ a uniformly closed subalgebra of $L(E)$ (though if $\mathfrak{A}$ is self-adjoint the answer is yes, since, by Kadison's Density Theorem (5), in this case topological irreducibility is equivalent to irreducibility). Since it is an open question whether the conclusion of Kadison's Theorem continues to hold when $\mathfrak{A}$ is not assumed selfadjoint, it is again difficult to construct a counter-example. 
We now return to the proof of Theorem 1, for which we need a slight generalisation of the construction used hitherto in this section. Suppose that $\mathfrak{A}$ is a normed algebra and that the $w^{*}$-closed absolutely convex set $C \subseteq \mathfrak{X}^{\prime}$ has polar $C^{\circ}$ in $\mathfrak{A}$. Then the operation of multiplication on the left by $a \in \mathfrak{A}$ induces a linear mapping $a_{c}$ on $\hat{\mathfrak{A}}_{c^{0}}$ if and only if $\bigcap_{\lambda>0} \lambda C^{\circ}$ is invariant under $a$, or equivalently $C a \subseteq \lambda C$ for some $\lambda>0$. When this is so we have (denoting the gauge of $C^{\circ}$ by $p_{c^{\circ}}$ )

$$
\begin{aligned}
\left\|a_{C}\right\| & =\sup \left\{p_{C^{\circ}}(a x): x \in C^{\circ}\right\} \\
& =\sup \left\{\lambda:|\langle x, y a\rangle|>\lambda \text { for some } x \in C^{\circ} \text { and } y \in C\right\} \\
& =\inf \left\{\lambda>0:|\langle x, y a\rangle| \leqq \lambda \text { for all } x \in C^{\circ} \text { and } y \in C\right\} \\
& =\inf \{\lambda>0: C a \subseteq \lambda C\} .
\end{aligned}
$$

When $C=\{h a:\|a\| \leqq 1\}$ this construction gives us the representation $a \rightarrow a_{h}$ of $\mathfrak{A}$ on $\mathfrak{U}_{h}$. When $h$ is weakly almost periodic but not autoperiodic the technique of (14) allows us to approximate the set $\{h a:\|a\| \leqq 1\}$ by autocompact sets, and so obtain a representation on a reflexive space.

Lemma 8. Let $W$ be an absolutely convex weakly compact set in a Banach space $E$. Then $W$ is the intersection of a decreasing sequence $\left(C_{m}\right)$ of autocompact sets each of which is invariant under every contraction in $\mathfrak{L}(E)$ leaving $W$ invariant.

Proof. For each $m \in N$ let $p_{n}^{m}$ be the gauge of $2^{n} W+(m n)^{-1} B$, where $B$ is the unit ball of $E$, and let $p^{m}(x)=\left\{3 \sum_{n=1}^{\infty} p_{n}^{m}(x)^{2}\right\}^{\frac{1}{2}}$ for each $x \in E$ (so that $p_{m}(x)$ may be infinite). It is shown in (14) that $C_{m}=\left\{x \in E: p^{m}(x) \leqq 1\right\}$ is autocompact, and it is easily seen that $W \subseteq C_{m}$ and that $C_{m}$ has the invariance property described in the enunciation. For fixed $n$ the sequence of sets $\left(2^{n} W+m^{-1} n^{-1} B\right)_{m \in N}$ is monotone decreasing and has intersection $2^{n} W$, so that $p_{n}^{m}(x) \uparrow 2^{-n} p_{W}(x)$ (where $p_{W}$ is the gauge of $W$ ) as $m \rightarrow \infty$ for every $x \in E$, even when the right-hand side is infinite. It follows that

$$
\lim _{m \rightarrow \infty} p^{m}(x)=\left\{3 \sum_{n=1}^{\infty} 2^{-2 n} p_{W}(x)^{2}\right\}^{\frac{1}{3}}=p_{W}(x)
$$

and consequently that $\bigcap C_{m}=W$.

Now consider a normed algebra $\mathfrak{A}$ and let $H$ be the set of weakly almost periodic functionals of unit norm on $\mathfrak{U}$. For the purposes of Theorem 1 we may assume that $\mathfrak{A}$ is complete and has an identity $e$ of unit norm, since if necessary we can adjoin such an identity and replace each $h \in H$ by $h \oplus 1$ : if $H$ is total or norm-generating then the same is true of the modified set with respect to the enlarged algebra.

For each $h \in H$ let $W_{h}$ be the norm-closure in $\mathfrak{A}^{\prime}$ of $\left\{h a:\|a\|_{\mathfrak{d}} \leqq 1\right\}$ and pick a sequence $\left(C_{h, m}\right)$ of autocompact sets whose intersection is $W_{h}$ as in 


\section{PERIODICITY OF FUNCTIONALS}

Lemma 8. The invariance property ensures that $C_{h, m} a \subseteq C_{h, m}$ whenever $\|a\|_{\mathfrak{a}} \leqq 1$, so the construction above associates with each $C_{h, m}$ a normreducing representation $a \rightarrow a_{h, m}$ of $\mathfrak{U}$ on a Banach space $E_{h, m}$; moreover, since $C_{h, m}$ is autocompact, the same is true of its polar in $\mathfrak{A}$-in other words, $E_{h, m}$ is reflexive. The $l^{2}$-sum $E$ of the spaces $E_{h, m}(h \in H, m \in N)$ is consequently reflexive. Corresponding to $a \in \mathfrak{U}$ define $T_{a} \in \mathfrak{L}(E)$ by

$$
T_{a}\left(x_{h, m}\right)=\left(a_{h, m} x_{h, m}\right) \text {. }
$$

Then $\left\|T_{a}\right\|=\sup _{h, m}\left\|a_{h, m}\right\| \leqq\|a\|_{\mathscr{e}}$, so that $T$ is a norm-reducing representation of $\mathfrak{A}$ on $E$.

To show that $T$ is actually isometric observe that, for any $h$ and $m$, if $C_{h, m} a \subseteq \lambda C_{h, m}$ then $h a \in \lambda C_{h, m}$ and so

and so

$$
|\langle a, h\rangle|=|\langle e, h a\rangle|=\lambda|\langle e, g\rangle| \text { for some } g \in C_{h, m},
$$

$$
\begin{aligned}
\left\|a_{h, m}\right\| & =\inf \left\{\lambda>0: C_{h, m} a \leqq \lambda C_{h, m}\right\} \\
& \geqq|h(a)| \inf \left\{|g(e)|^{-1}: g \in C_{h, m}\right\} .
\end{aligned}
$$

Fix $h \in H$ and pick $g_{m} \in C_{h, m}$ such that

$$
\left|g_{m}(e)\right| \geqq(1-1 / m) \sup \left\{|g(e)|: g \in C_{h, m}\right\} \text {. }
$$

The sequence $\left(g_{m}\right)$ has a $w^{*}$-cluster point $g_{0} \in \bigcap_{m} C_{h, m}=W_{h}$, and so

$$
\begin{aligned}
\limsup _{m \rightarrow \infty}\left\|a_{h, m}\right\| & \geqq|h(a)|\left|g_{0}(e)\right|^{-1} \\
& \geqq|h(a)| \inf _{g \in W_{h}}|g(e)|^{-1} \\
& =|h(a)| \inf _{\|b\| \leqq 1}|h b(e)|^{-1} \\
& =|h(a)| /\|h\|=|h(a)| .
\end{aligned}
$$

Thus $\left\|T_{a}\right\| \geqq \sup _{h \in H}|h(a)|$, so that if $H$ is total then $T$ is faithful and if $H$ is norm-generating then $T$ is isometric.

\section{REFERENCES}

(1) B. A. BARNES, Representations of normed algebras with minimal left ideals, Proc. Edinburgh Math. Soc. 19 (1974), 173-190.

(2) E. Berkson and H. Porta, Representations of $\mathfrak{B}(X)$, J. Functional Analysis 3 (1969), 1-34.

(3) F. F. Bonsall and J. DunCan, Complete normed algebras (Springer Verlag, Berlin, 1974).

(4) P. Crvin and B. Yood, The second conjugate space of a Banach algebra as an algebra, Pacific J. Math. 11 (1961), 847-870. 
(5) J. DixmIER, Les $C^{*}$-algèbres et leurs représentations (Gauthier-Villars, Paris, 1964).

(6) A. Grothendieck, Produits tensoriels topologiques et espaces nucléaires, Mem. Amer. Math. Soc. 16 (1955).

(7) A. Grothendieck, Critères de compacité dans les espaces fonctionnels généraux, Amer. J. Math. 74 (1952), 168-186.

(8) H. Halpern, Finite sums of irreducible functionals on $C^{*}$-algebras, Proc. Amer. Math. Soc. 18 (1967), 352-358.

(9) B. E. Johnson, The uniqueness of the (complete) norm topology, Bull. Amer. Math. Soc. 73 (1967), 537-539.

(10) J. S. PYM, The convolution of functionals on spaces of bounded functions, Proc. London Math. Soc. (3) 15 (1965), 84-104.

(11) A. P. ROBERTSON and W. RoBertson, Topological vector spaces (Cambridge University Press, 1964).

(12) N. J. Young, Separate continuity and multilinear operations, Proc. London Math. Soc. (3) 26 (1973), 289-319.

(13) N. J. Young, The irregularity of multiplication in group algebras, Quarterly J. Math. (2) 24 (1973), 59-62.

(14) W. J. Davis, T. Figiel, W. B. Johnson and A. Pelczyński, Factoring weakly compact operators, J. Functional Analysis 17 (1974), 311-327.

THE UNIVERSITY

Glasgow G12 8QW 